THE KURUME MEDICAL JOURNAL Vol. 17, No. 3, 1970

\title{
EXPERIENCES ON POSITIVE PRESSURE PNEUMOVENTRICULO. GRAPHY FOR STEREOTAXIC SURGERY
}

\author{
SHINKEN KURAMOTO AND MITSUO WATANABE \\ Neurosurgical Clinic, Kurume University Hospital, Kurume, Japan
}

(Received for publication April 30, 1970)

\section{INTRODUCTION}

To select the most effective target point at stereotaxic surgery and confine the destruction to the minimum is the best way to obtain satisfactory operative results without having any complication. This requires in the first place exact visualization of the third ventricle in order to take an anatomically accurate measurement of it. And then, it is necessary that visualization of the third ventricle should be performed with patients kept supine in the stereotaxic apparatus without changing their position. We attempted positive pressure ventriculography described by Calvin and $\mathrm{Coe}^{1 \text { ) }}$ in 1967 and obtained satisfactory results so that they are reported.

\section{MATERIAL}

In 20 cases undergoing the stereotaxic operation at our clinic during a period of from November 1968 to August 1969, positive pressure pneumoventriculography (PVG) was conducted. These 20 cases consisted of 14 cases of Parkinsonism, 2 cases of Huntington's chorea, 2 cases of behavior disorder with epilepsy, and 2 cases of torsion dystonia. The age varied between 16 and 72 years, and 3 cases were operated upon under general anesthesia and the remaining 17 under local anesthesia. The operative results obtained were all but one case of torsion dystonia satisfactory once without having any complications.

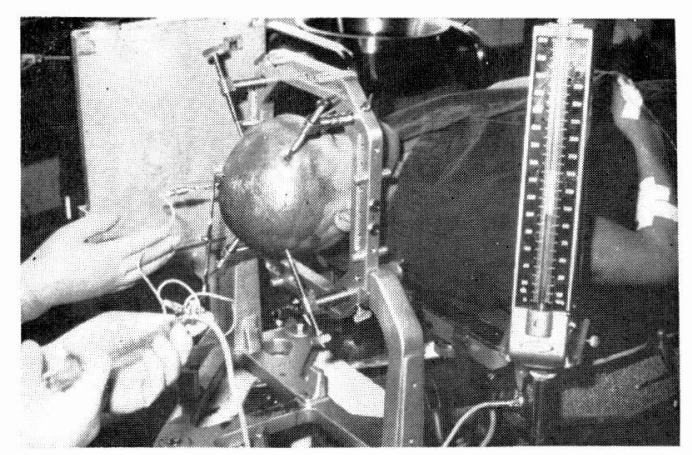

Fig. 1. Positive pressure pneumoventriculography at the stereotaxic operation. 


\section{METHOD}

The patient was placed supine in the stereotaxic apparatus and a frontal burr hole was made. From there, the vicinity of the Foramen Monroi of a lateral ventricle was punctured by means of an 18-gauge ventricular needle, and then air-fluid replacement was carried out under atmospheric pressure. Regarding the apparatus measuring intraventricular pressure, connecters were fixed to both ends of a vinyl tube and a three-way stopeock was inserted into the middle of the tube. One connecter was joined with a punctured needle and the other with a mercury manometer, and a $20 \mathrm{cc}$ lock syringe was connected with a three-way stopcock. The connecters of the puncture needle and manometer were then opened, and while air was being injected through a syringe into the ventricle slowly at a speed of 1 ce per 2 and 3 seconds, the intraventricular pressure might be continuously read by mercury manometer.

In the first 5 cases the intraventricular pressure used as an index was raised up to $35-40 \mathrm{mmHg}$ and roentgenographies were taken. In other 15 cases roentgenographies were taken before injection and at every injection of 5 ec of air in order to estimate the amount of visualization. The total amount of air injected was 20-30 ce. After the completion of roentgenography, the air in the ventricle was removed gradually till the inner pressure was reduced to the atmospheric one.

The time assigned for these procedures amounted to about 20-30 minutes and the duration of increasing pressure was within 4 minutes.

\section{RESULTS}
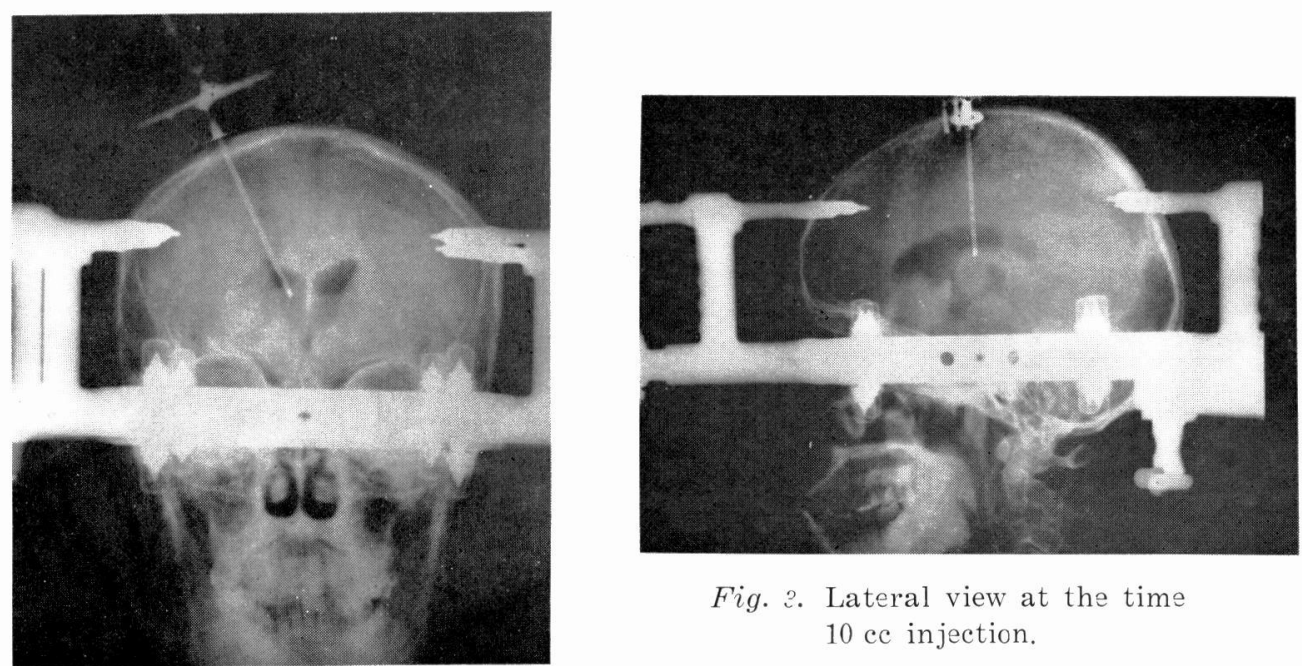

Fig. ?. Lateral view at the time 10 ec injection.

Fig. 2. Frontal view at the time 10 ec injection. 
Positive pressure PVG in the 20 cases resulted in clear visualization of the frontal part of lateral ventricles on both sides, the third and fourth ventricles. Especially the entire third ventricle was distinctly visualized (Fig. 2 and 3).

The air introduction with the intraventricular pressure rising up to $35-40$ $\mathrm{mmHg}$ attempted in the first 5 cases revealed that the relationship between the injected amount of air and the intraventricular pressure differed case by case, especially depending on the degree of the ventricular dilation existing.

Secondly, on cases with no or little dilatation of the ventricle and on those with its moderate to high-degree dilatation, changes in the intraventricular pressure were pursued in relation to the injected amount of air, as given in Tables 1 and 2, and at the same time, the progress of visualization of the

TABLE 1

The injected amount of air (cc) and intraventricular pressure ( $\mathrm{mmHg}$ ) (Cases of the undilated ventricle)

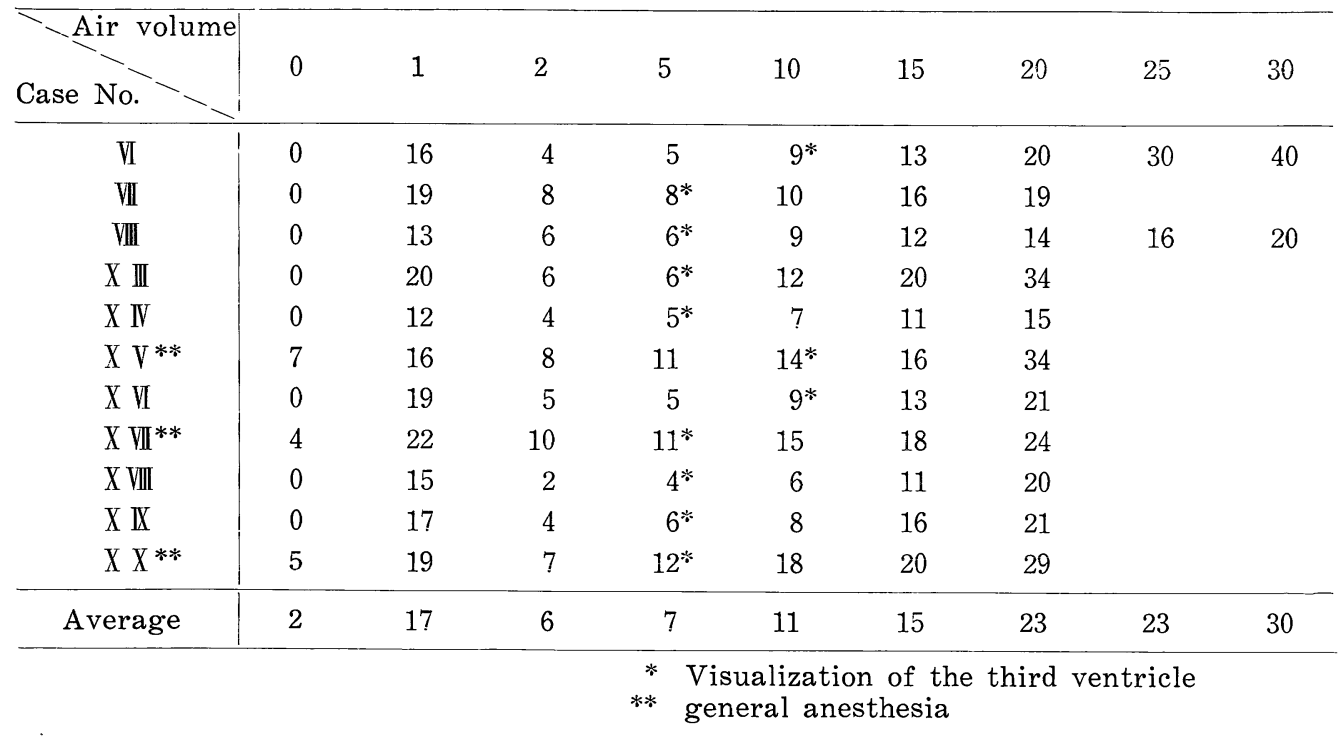

TABLE 2

The injected amount of air (cc) and intraventricular pressure ( $m m H g)$ (Cases of the dilated ventricle)

\begin{tabular}{c|ccccccccc}
\hline Air volume & 0 & 1 & 2 & 5 & 10 & 15 & 20 & 25 & 30 \\
\hline Case No. & 0 & & & & & & & \\
\hline X & 0 & 14 & 22 & 6 & 3 & $5^{*}$ & 8 & 13 & 34 \\
X & 0 & 20 & 12 & 8 & 8 & $9^{*}$ & 13 & 18 & 23 \\
XI & 0 & 20 & 10 & 9 & $9^{*}$ & 11 & 11 & 13 & 16 \\
XII & 0 & 18 & 16 & 7 & 7 & $7^{*}$ & 10 & 12 & 18 \\
\hline Average & 0 & 18 & 15 & 8 & 7 & 8 & 11 & 14 & 23 \\
\hline
\end{tabular}

* Visualization of the third ventricle 
ventricular system was also examined by roentgenography made at every time of injection of $5 \mathrm{cc}$. From this, it was found that the intraventricular pressure swung upward rapidly under air introduction of $1-2$ ec in all instances, and then also went down rapidly, although in cases of the dilated ventricle the drop of pressure was delayed a little. In cases of the non-dilated ventricle, an additional introduction of air made the pressure increased again at $10 \mathrm{cc}$ injection and reached average $23 \mathrm{mmHg}$ at $20 \mathrm{cc}$ injection, while in cases of the dilated ventricle the pressure began to increase at the time of 20 cc injection and reached $23 \mathrm{mmHg}$ at $30 \mathrm{cc}$ injection. In short, in the latter cases the increase in pressure occured later and its degree was slight.

As shown in Tables 1 and 2, in cases of the non-dilated ventricle the time of visualizing the entire third ventricle coincided with the time of 5-10 cc injection and the intraventricular pressure at that time was 4-18 $\mathrm{mmHg}$, whereas in cases of the dilated ventricle the third ventricle could be visualized entirely by the time of $10-15$ cc injection with an intraventricular pressure of 5-9 $\mathrm{mmHg}$. Under general anesthesia, however, the pressure was generally higher than under local anesthesia. In addition, when an additional air was introduced, the air was displaced from the fourth ventricle into the subarachnoid space of the basilar cisterns and further into the frontal subarachnoid space.

As regards the side effects a slight headache was noted in 10 of 20 cases, 2 cases of which were associated with nausea, but these symptom disappeared immediately after release from pressure. They appeared when $16-25 \mathrm{cc}$ of air was injected under intraventricular pressure of $11-30 \mathrm{mmHg}$, and the time of their manifestation corresponded to that when the air was displaced into the subarachnoid space on roentgenograms.

\section{DISCUSSION}

The purpose of visualizing the ventricular system for stereotaxic surgery is to delineate the anatomical structures of the ventricular system including the third ventricle in detail and get thereby on exact measuring point. Moreover, since the stereotaxic surgery is performed under local anesthesia without changing the position of supine patients in the stereotaxic apparatus, it must be completed in a possible short time without giving any discomfort to the patients.

Although at present pneumoencephalography or pneumoventriculography is generally performed sometimes when visualization of the third ventricle is insufficient, and then positive contrast ventriculography is conducted. Positive contrast ventriculography was first developed by Sicard et al. in $1923^{3)}$, and when Bull et al. (1950) ${ }^{4}$ used Pantopaque as a contrast medium it came to be widely utilized for diagnosis. At stereotaxic surgery, Sedzimir (1962) 5) and Vinas (1964) ${ }^{6}$ inserted a small catheter through the lateral ventricle into the third ventricle, through which a positive contrast medium was injected. 
However, as this method is complicated in manipulation and it is sometimes difficult to insert the catheter into the third ventricle, or owing to the fact that a positive contrast medium has a property to irritate the central nervous tissue, it cannot in general be used.

On the other hand, Clark et al. (1966) ${ }^{7}$ ) detained a small catheter in the lumbar subarachnoid space providing, at the same time, the lateral ventricle with a drainage, and by inserting a cylinder into it a closed circuit was made outside the body. Thus, he devised a method to visualize the ventricular system using a small quantity of air and afterward withdraw the air injected into the cylinder by means of the up-and-down movement of the saline solution and air contained in the cylinder. This method excels in that a small amount of air introduced is sufficient enough to visualize the ventriclar system with the patient kept in the intraventricular pressure. Nevertheless, it has some disadvantages, for instance it is trouble-some in detaining a catheter in the lumbar subarachnoid space and liable to be attended with danger of infection.

As a procedure to visualize the third ventricle without changing the positive of supine patients. Calvin and Coe ${ }^{1)}$ performed positive pressure ventriculography in 45 cases and reported successesful visualization of the third ventricle in the entire cases producing few side effects. They described that at roentgenography done during the time of positive pressure injection of $20 \mathrm{cc}$ of air into the lateral ventricle the intraventricular pressure proved to be $20-25$ $\mathrm{mmHg}$.

We also carried out positive pressure pneumoventriculography in 20 cases in almost the same way as that of Calvin's et al., and investigated in detail the changes occurring in the intraventricular pressure in connection with the amount of air injected under increasing pressure. As a result, it was found that the relation of the injected amount to the pressure varies between cases of the dilated ventricle and those of the undilated one. However, the general tendency in both cases was found to be the same, that is, by injection of air, the air in the lateral ventricle on the injected side is displaced rapidly into the lateral ventricle on the opposite side, and almost at the same time there occur a rapid rise and decline of intraventricular pressure. And then, after it continues to remain constant for a while, the pressure rises gradually. It is characteristic of cases of the dilated ventricle that a rise of pressure is delayed and its gradient is gentle.

In cases of the undilated ventricle, the injected amount of air necessary for visualizing the entire third ventricle was $10 \mathrm{cc}$, the pressure at that time being 6-18 $\mathrm{mmHg}$, while in cases of the dilated ventricle $15 \mathrm{cc}$ of air was necessary and the pressure at that time was 5-11 mmHg. Although 10-15 ce of air injected is sufficient enough for actual positive pressure PVG, it may be used as an index when air is escaped from around the ventricular needle at the determination of the intraventricular pressure during injection. Since a slight headache and nausea were observed sometimes as side effects of this procedure 
and disappeared immediately after release from pressure, there was no trouble in performing stereotaxic surgery under local anesthesia.

In expectation of the dilatation of the ventricular system due to increasing intraventricular pressure, pneumoencephalography was conducted in all the cases traced preoperatively and the ventricular system was measured. The results of measurement was compared with those obtained under increasing pressure consequently, it was confirmed that in cases of the dilated ventricle, as seen in the relationship between the injected amount of air and intraventricular pressure, the edge of the lateral ventricle in some cases was made roundish and appeared to be distended. As to the third ventricle, in cases of the dilated ventricle it sometimes showed a slight increase in width when the intraventricular pressure exceeded $30 \mathrm{mmHg}$, but its increase in width was under $0.5 \mathrm{~mm}$, and there was no change in the CA-CP line. Accordingly, it may be assumed that the pressure of $5-18 \mathrm{mmHg}$ capable of visualizing the third ventricle completely cannot cause the dilatation of the ventricle, so that it is no trouble in the measurement at stereotaxic operation.

\section{CONCLUSION}

For the purpose of visualizing the third ventricle for stereotaxic surgery, positive pressure pneumoventriculography was performed in 20 cases and the following satisfactory results were obtained:

1) On all occasions, the third ventricle was visualized completed by positive pressure pneumoventriculography without changing the position of supine patients at stereotaxic surgery.

2) Ten or 15 cc of air introduced into the lateral ventricle was sufficient enough to visualize the third ventricle and the intraventricular pressure at that time was under $18 \mathrm{mmHg}$.

3) In cases of the dilated ventricle, the increase of pressure due to air introduction is delayed and its gradient is gentle as compared with cases of the non-dilated ventricle.

4) The dilatation of the ventricle due to air introduction is very slight, which exerts no influence on stereotaxic surgery.

5) Side effects due to increasing pressure were all mild and disappeared immediately after release from pressure.

\section{REFERENCES}

1) Calvin, T. H. and CoE, J. E.: Positive pressure ventriculography for stereotaxic surgery. J. Neurosurg. 26, 548-552, 1967.

2) Ken-ichiro, Sugita: New stereotaxic apparatus and high frequency coagulation with thermistor. Brain and Nerve, 18, 177-187, 1966.

3) Sicard, J. A., Paraf, J. and Laplane, L. : Radiodiagnostic rachidien lipiodole'. 
Ponction atloidooccipitale et cervicale. Pr. med., 31, 885-887, 1923.

4) Bull, J. W. D. : Positive contrast ventriculography. Acta radiol., Stockh, 34, 253268, 1950.

5) Sedzimir, C. B. and Iwan, S. R. : Simplified contrast ventriculography. J. Neurosurg., 19, 657-660, 1962.

6) Vinas, F. J. : Iodoventriculography by direct of childhood. J. Neurosurg. 21, 492496, 1964.

7) Clark, L., Moser, D. and Brackett, C. : Controlled small volume ventriculography. J. Neurosurg., 24, 777-778, 1966. 